\title{
Interplay between Exosomes and Autophagy in Cardiovascular Diseases: Novel Promising Target for Diagnostic and Therapeutic Application
}

\author{
Jinfan Tian ${ }^{1,2}$, Mohammad Sharif Popal', Yingke Zhao³, Yanfei Liu', Keji Chen², Yue Liu, ${ }^{2}$, \\ ${ }^{1}$ Department of Cardiology, Beijing Anzhen Hospital, Capital Medical University, Beijing, China \\ ${ }^{2}$ Cardiovascular disease center, Xiyuan Hospital, China Academy of Chinese Medical Sciences, Beijing, China \\ ${ }^{3} \mathrm{Li}$ Ka Shing Faculty of Medicine, The University of HongKong, Pokfulam, Hong Kong. \\ ${ }^{4}$ Graduate School, Beijing University of Chinese Medicine, Beijing, China
}

[Received September 12, 2018; Revised October 14, 2018; Accepted October 20, 2018]

\begin{abstract}
Exosome, is identified as a nature nanocarrier and intercellular messenger that regulates cell to cell communication. Autophagy is critical in maintenance of protein homeostasis by degradation of damaged proteins and organelles. Autophagy and exosomes take pivotal roles in cellular homeostasis and cardiovascular disease. Currently, the coordinated mechanisms for exosomes and autophagy in the maintenance of cellular fitness are now garnering much attention. In the present review, we discussed the interplay of exosomes and autophagy in the context of physiology and pathology of the heart, which might provide novel insights for diagnostic and therapeutic application of cardiovascular diseases.
\end{abstract}

Key words: exosomes, autophagy, cardiovascular disease, atherosclerosis, myocardial ischemia/reperfusion injury

\section{Introduction}

Currently, it has been established that the messenger functions of exosomes are critical in neointimal formation, vascular repair, and atherosclerosis [1-3]. Moderate autophagy exerts protective role in cardiovascular diseases (CVDs)[4, 5]. Evidence has disclosed that the existence of a crosstalk between exosomes and autophagy in the protection of cellular homeostasis [6-8]. Exosomes contain molecular signals that regulate autophagy, which in turn, regulate exosomal activities $[6,9,10]$.

Extracellular vesicles like micro-vesicles and apoptotic bodies serve as intercellular messengers. The early endosomes were generated from plasma membrane through inward of buds under the stimulation of physical and or chemical factors. The late endosomal membrane inward buds and produces multivesicular bodies named MVBs, directed by the machinery of endosomal sorting complex required for transport (ESCRT), eventually leading to the accumulation of intraluminal vesicles (ILVs) within MVBs [1]. MVBs can either fuse with lysosomes, which subsequently fuse with autophagosome to form autolysosome leading to autophagic degradation, or fuse with the plasma membrane to release vesicles, such as exosomes, into the extracellular space [6] (Fig. 1).

*Correspondence should be addressed to: Dr. Yue Liu, Cardiovascular disease center, Xiyuan Hospital of China Academy of Chinese Medical Sciences, Xiyuan caochang road 1\#, Beijing 100091, China. Email: liuyueheart@ hotmail.com.

Copyright: @ 2018 Tian J et al. This is an open-access article distributed under the terms of the Creative Commons Attribution License, which permits unrestricted use, distribution, and reproduction in any medium, provided the original author and source are credited. 


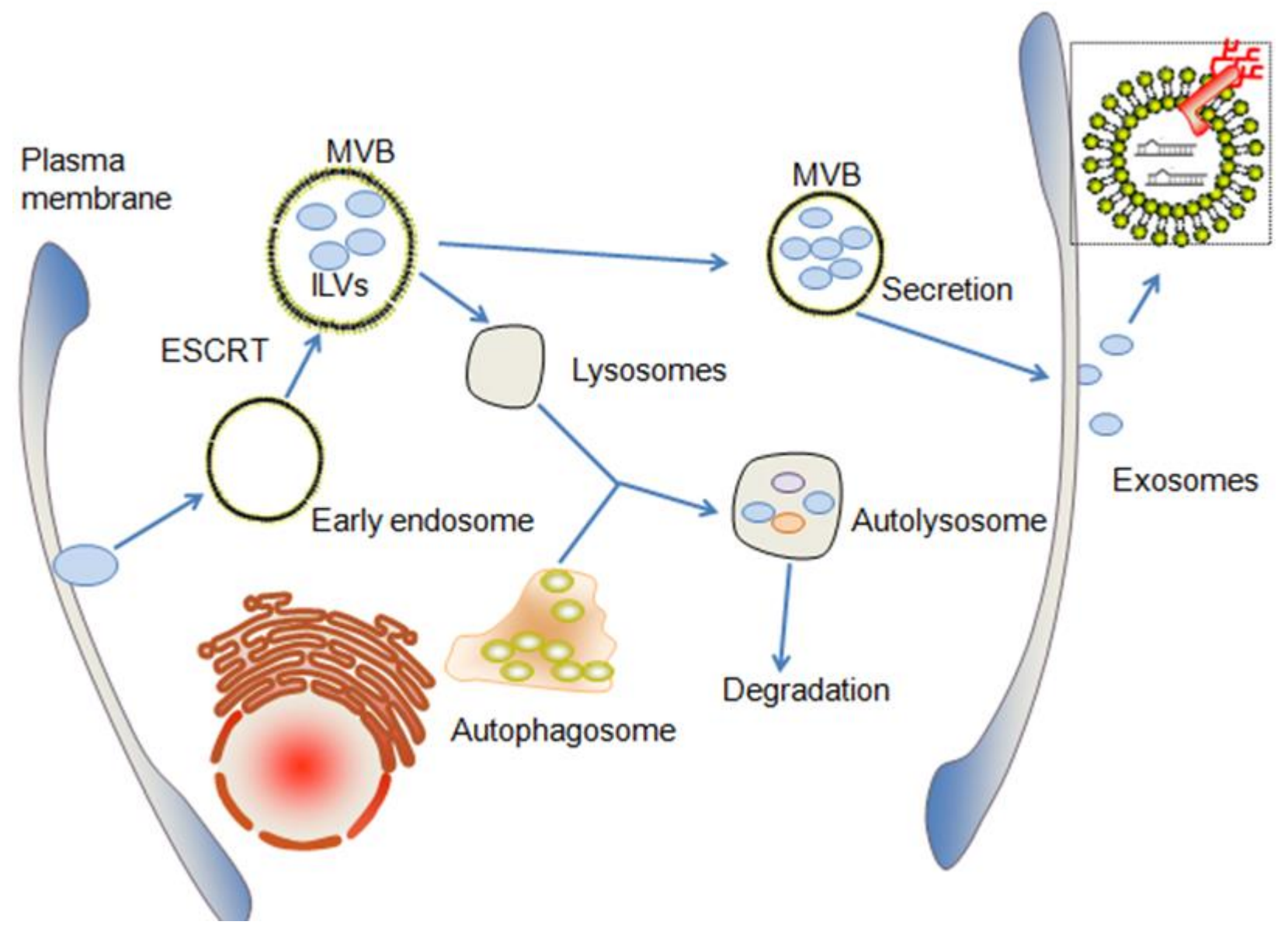

Figure 1. The crosstalk between autophagic and exosomal process. MVBs which enrich in ILVs generates from early endosomes directed by ESCRT. MVBs can either fuse with lysosomes, which subsequently fuse with auto-phagosome to form autolysosome leading to autophagic degradation, or MVBs could fuse with the plasma membrane to release vesicles as exosomes into the extracellular space. Exosomes contains rich biological signaling materials as non-coding RNAs. ESCRT, endosomal sorting complex required for transport; ILVs, intraluminal vesicles.

The endosomal and autophagic procedures are consequences of the biogenesis of exosomes which depend on the cellular activity of autophagy $[6,7]$. The suppression of autophagosome maturation or fusion with a lysosome commonly results in inadequate digestion of damaged proteins or organelles, leading to progressive accumulation of deleterious material, might promote exosomal procedure to release the partially digested or undigested materials $[6,7,10]$. Alternatively, under cellular starvation conditions, the bio-balance would be shifted towards greater autophagic degradation, and a reduced biogenesis of exosomes [6, 7, 10]. Taken together, induction of the autophagy pathway has been shown to decrease exosomal release by promoting fusion of MVBs with autophagosomes, while impaired autophagy may lead to increase exosomes secretion. ILVs enrich in biological materials, including messenger RNA
(mRNA), microRNA (miRNA), and small interfering RNA (siRNA). Exosomes transfer cell-cell communication and capable of regulating autophagy by these biological molecules $[11,12]$. This review for the first time discussed the interplay of exosomes, autophagy and CVDs.

\section{Exosomes-related non-coding RNAs and CVDs}

The roles of exosomes in CVDs meticulously depend on the type of cell it is derived from $[1,13]$. Correspondingly, exosomes-related non-coding RNAs exert various functions in the progression of CVDs [14] (Table 1). 
Table 1. Overview of exosomes-related non-coding RNAs in cardiovascular disease

\begin{tabular}{|c|c|c|c|}
\hline non-coding RNA & Cardiovascular disease & Cell types & Effects \\
\hline IncRNA GAS5 & Atherosclerosis $\uparrow$ & THP-1 cells & $\begin{array}{l}\text { The apoptosis of vascular endothelia cells } \uparrow \\
\text { [16] }\end{array}$ \\
\hline $\operatorname{miR}-143 / 145$ & Atherosclerosis $\downarrow$ & Endothelial cells & $\begin{array}{l}\text { Communication between endothelial and } \\
\text { smooth cells } \uparrow[17]\end{array}$ \\
\hline miR-155 & Atherosclerosis $\uparrow$ & Vascular smooth muscles cells & Endothelial permeability $\uparrow[18]$ \\
\hline $\begin{array}{l}\text { miR-223, miR-339 } \\
\text { and miR-21 }\end{array}$ & Atherosclerosis $\downarrow$ & Platelet & NF- $\kappa B$ pathways $\downarrow[19,20]$ \\
\hline miR-21 & Myocardial infarction $\downarrow$ & EnMSCs & Cardiomyocytes apoptosis $\downarrow$ [36] \\
\hline miR-126 & $\begin{array}{l}\text { Myocardial } \\
\text { ischemia/reperfusion injury } \downarrow\end{array}$ & Adipose-derived stem cells & $\begin{array}{l}\text { Apoptosis, inflammation, } \\
\text { fibrosis } \downarrow \text { angiogenesis } \uparrow \text { [37] }\end{array}$ \\
\hline miR-93-5p & Myocardial infarction $\downarrow$ & Adipose-derived stromal cells & $\begin{array}{l}\text { Hypoxia-induced autophagy and } \\
\text { inflammation } \downarrow[11]\end{array}$ \\
\hline $\operatorname{miR}-451$ & $\begin{array}{l}\text { Myocardial ischemia/ } \\
\text { reperfusion injury } \downarrow\end{array}$ & Cardiac progenitor cells & Oxidative stress-induced apoptosis $\downarrow[40]$ \\
\hline $\operatorname{miR}-21$ & $\begin{array}{l}\text { Myocardial } \\
\text { ischemia/reperfusion injury } \downarrow\end{array}$ & Cardiac progenitor cells & Oxidative stress-induced apoptosis $\downarrow[41]$ \\
\hline
\end{tabular}

\subsection{Exosomes-related non-coding RNAs and atherosclerosis}

It has been established that atherosclerosis is characterized by ox-LDL induced inflammation, endothelial injury, apoptosis and phonotype changes of vascular cells and platelet activation. Most recent studies demonstrated that, the exosomal pathway for communication of macrophages and vascular endothelial cells modulates the apoptosis and inflammation response, participating in the pathophysiology of atherosclerosis [3, $14,15,16]$. Furthermore, the communication between endothelial cells and smooth muscle cells mediated by exosomes affects the inflammation and phonotype changes of vascular cells, which are closely associated with the progression of arthrosclerosis [17]. Huang C et $a l$. [15] revealed that, inhibiting the secretion of exosomes by ox-LDL-stimulated macrophages restored the growth and tube formation of endothelial cells. The findings of Chen L et al. [16] revealed that exosomes derived from IncRNA GAS5 over-expressed THP-1 cells enhanced the apoptosis of vascular endothelia cells, suggesting that exosomes-associated IncRNA GAS5 regulated the apoptosis of macrophages and endothelial cells. According to Eduard Hergenreider $\mathrm{E}$ et al. [17], extracellular vesicles derived from KLF2-expressing endothelial cells which enrich in miR-143/145 reduced atherosclerotic lesion formation in the aorta of ApoE-/mice by increased communication between endothelia cells and smooth muscle cells through miRNA- and extracellular-mediated mechanism. Study from Zheng B et al. [18] revealed that the transfer of Krüppel-like factor 5 (KLF5)-induced miR-155 from Smooth muscle cells
(SMCs) to endothelial cells mediated by vascular smooth muscle cells (VSMCs)-derived exosomes, contributed to impaired tight junctions and the integrity of endothelia barriers, leading to the increased endothelial permeability and enhancing atherosclerotic progress.

Platelet activation and endothelial damage take pivotal roles in atherosclerosis. The findings of $\mathrm{Li} \mathrm{J}$ et al. [19] revealed that the levels of miR-223, miR-339 and miR-21 were elevated in thrombin-activated plateletderived exosomes. The expression of intercellular adhesion molecule-1 (ICAM-1) stimulated by Tumor necrosis factor- $\alpha$ (TNF- $\alpha$ ) in human umbilical vein endothelia cells (HUVECs) was significantly inhibited by miR-223 transfection via inhibition of the phosphorylation of $\mathrm{p} 38$ mitogen-activated protein kinase (MAPK), c-Jun N-terminal kinase (JNK) and extracellular regulated protein kinases (ERK), and downregulation of NF- $\mathrm{B}$ pathway. The finding suggested that thrombin-activated platelet-derived exosomes exerted protective effects against atherosclerosis and endothelial inflammation. The downregulating effect of exosomes on ICAM-1 expression was reversed by miR-223 inhibitor. Tan $\mathrm{M}$ et al. [20] verified that platelet-derived exosomes containing miR-223, miR-339 and miR-21 could be transferred into smooth muscle cells, besides that, it can inhibit plateletderived growth-factor receptor-beta-stimulated smooth muscle cells proliferation. These findings provide evidence that platelet-derived exosomes could be a promising target for atherosclerosis.

Recently, other types of cells are also reported to be closely related to atherosclerosis. Insulin resistance adipocyte-derived exosomes has been shown to promote 
plaque burden and plaque vulnerability partly by inducing vasa vasorum angiogenesis in diabetic ApoE-/- mice [21]. Gao W et al. [22] observed that mature dendritic cellsexosomes increased HUVECs inflammation via NF- $\mathrm{kB}$ pathway in vivo, they further found that the atherosclerotic lesions in ApoE-/- mice was significantly increased by a period of mature dendritic cells injection into ApoE-/- mice. These findings provide novel targets for the management of atherosclerosis.

\subsection{Exosomes-related non-coding RNAs in acute myocardial infarction}

Evidence showed that plasma levels of exosomes-related miRNAs could serve as a novel biomarker for diagnosis of acute myocardial infarction (AMI) [23, 24]. According to Corsten MF et al. [25] circulating miRNA-208b were extremely elevated in plasma among AMI patients. Interestingly, miR-499-5p was reported decreased in the infracted area of rat heart, whereas the plasma level was elevated both in AMI rats and patients [26, 27]. Thus, serum miR-449-5p serves as a biomarker for diagnosis of AMI [26-28]. Yang Y et al. [29] found that the level of miR-30a increased in the serum of AMI patients in a timedependent manner. Relative increase of circulating miR$122-5 p$, as reflected by a high $\mathrm{miR}-122-5 \mathrm{p} / 133 \mathrm{~b}$ ratio, is a predictive biomarker to identify patients who at higher risk of an adverse prognosis after ST-elevation myocardial infarction (STEMI). The power of early prognostic value is increased when evaluated in association with left ventricular ejection fraction (LVEF)[30].

Furthermore, preclinical studies also revealed Mesenchymal stem cell (MSC)-derived exosomes exerts beneficial effects for the AMI treatment [31, 32]. MSCexosomes improved cardiac function by stimulating neovascularization and inhibiting the inflammation response [33]. Arslan F et al. [31] showed that bone marrow MSC-derived exosomes reduced infarct size, enhanced myocardial viability and prevented adverse remodeling after ischemic/reperfusion injury in SpragueDawley rats via activation of phosphatidylinositol 3kinase (PI3K)-Akt pathway. According to Zhang Z et al. [34], bone marrow MSC-exosomes, containing a large number of bioactive compounds, stimulated the proliferation and angiogenic potency of cardiac stem cells (CSCs). In a rat myocardial infarction model, bone marrow MSC-derived exosomespreconditioned CSCs increased survival, enhanced capillary density, reduced cardiac fibrosis and restored long-term cardiac function. Wang XL et al. [35] revealed that, exosomes derived from human umbilical cord mensenchymal stem cells increased myocardial repair in Sprague-Dawley rats by promoting Smad 7 expression via inhibiting miR-125b-5p. KAN WANG et al. [36] for the first time reported that the superiority of MSCs derived from the endometirum (EnMSCs) in anti-MI therapy over those derived from bone marrow or adipose tissue. Exosomal miR-21 that releases from EnMSCs ameliorates cardiac function by regulating cell apoptosis and angiogenesis, and the benefits could be abolished through knockdown of miR-21. These results indicate that MSC-derived exosomes are a potential candidate for adjunctive therapy for patients suffering from acute myocardial infarction.

In addition, the therapeutic value of other type cells derived exosomes for myocardial infarction and the involved mechanisms were investigated in the recent years. According to Luo Q et al. [37], exosomes from miR-126-overexpressing adipose-derived stem cells prevented myocardial damage in a rat model via inhibiting apoptosis, inflammation, fibrosis and increased angiogenesis. Adipose-derived stromal cells (ADSC)derived miR-93-5p-containging exosomes attenuates the infarction-induced rat myocardial damage by inhibition hypoxia-induced autophagy and inflammation through targeting Atg7 and TLR4 respectively [11]. Another study also revealed that miR-145-5p protects $\mathrm{H} 9 \mathrm{C} 2$ cardiac cells from hypoxia-induced inflammatory response and apoptosis [38]. V Vicencio JM et al. [39] showed that plasma exosomes protect the myocardium from ischemiareperfusion injury via a HSP70/Toll-like receptor 4 (TLR4) communication pro-survival signaling. Cardiac progenitor (CPC)-derived exosomes emerged as one of the targets for cardiac protection. Chen L et al. [40] for the first time showed that cardiac progenitor-derived exosomes (CPC-exosomes), which maintains a high-level expression of GATA4-responsive-miR-451, protected H9C2 myocardial cells from oxidative stress by inhibiting caspase 3/7 activation in vitro. Furthermore, CPCexosomes inhibited cardiomyocyte apoptosis by about $53 \%$ compared to PBS control in an acute mouse myocardial ischemia/reperfusion model. Xiao J et al. [41] reported that, CPC-exosomes miR-21 played an inhibitory role in the apoptosis pathway through downregulating programmed cell death 4 (PDCD4). Restored miR-21/PDCD4 pathway utilizing CPC-derived exosomes could protect $\mathrm{H} 9 \mathrm{C} 2$ cells against oxidative stress-related apoptosis.

\subsection{Exosomes-related non-coding RNAs and Cardiomyopathy}

Myocardial fibrosis and remodeling are pathophysiological mechanisms for cardiomyopathy. De Gonzalo-Calvo D et al. [42] first demonstrated that serum levels of cardiomyocyte-enriched miRNA-1 and miR$133 \mathrm{a}$ independently predict myocardial steatosis in 
patients with well-controlled and uncomplicated type 2 diabetes of short duration. Wang X et al. [43] disclosed that elevation of $\mathrm{Hsp} 20$ in cardiomyocytes offer protection in diabetic mice hearts through the release of instrumental exosomes.

Table 2. Exosomes-related miRNAs regulate ABCA1.

\begin{tabular}{ll}
\hline miRNA & Effects \\
\hline miR-33 & ABCA1 $\downarrow[56-59]$ \\
miR-758 & ABCA1 $\downarrow[61]$ \\
miR-20a/b & ABCA1 $[62]$ \\
miR-19b & ABCA1 [63] \\
miRNA-302a & ABCA1 $\downarrow[64]$ \\
\hline ABCA1, ATP-binding cassette transporter A1
\end{tabular}

\section{Exosomes-related miRNA regulates Autophagy in CVDs}

Autophagy is an intracellular metabolic self-degradative process regulated by more than 30 highly conserved autophagy-related genes (ATG), in which unwanted cytoplasmic proteins and damaged organelles are degraded by lysosomes. Moderate autophagy is essential for cell homeostasis, while excess of autophagy triggers cell death, indicating that autophagy has both the protective and detrimental functions in the pathological settings $[4,44,45]$. Autophagy is established as a doubleedged sword in CVDs [4, 46]. MiRNAs have been exposed to regulate autophagy through posttranscriptional repression of Atg or upstream effectors (BECN1, mTOR, and ULK1) [47, 48]. Vulnerable plaques are characterized by lipid-filled necrotic core resulted from accumulation of apoptotic cells and defective phagocytic clearance [49]. Accumulating evidence supported that macrophages autophagy is an emerging target for atherosclerosis [4, 50-53]. The protective function of autophagy against atherosclerosis associated with cholesterol metabolism and lipophagy. Autophagy facilitates macrophage cholesterol efflux mediated by an ATP-binding cassette transporter A1 (ABCA1)-dependent pathway [54]. The inducing of autophagy via Adenosine 5'-monophosphate (AMP)activated protein kinase (AMPK) activation is one of the mechanisms by which statins lower plasma cholesterol [55]. Collectively, exosomes-related miRNA regulates autophagy and cholesterol efflux by modulating the expression of ABCA1 (Table 2). MiR-33 represses the expression of $A B C A 1$, reduces cholesterol efflux. On the contrary, inhibition of miR-33 enhances ABCA1 expression, and circulating HDL levels, and improves cholesterol efflux from macrophages, leading to attenuation of atherosclerosis [56-59]. Ouimet $\mathrm{M}$ et al. [60] provided an insight into the mechanism by which miRNA regulates cellular cholesterol homeostasis and atherosclerosis. The study showed that macrophages treated with anti-miRNA-33 increased efferocytosis, lysosomal biogenesis, and degradation of apoptotic material clearance. Treating atherosclerotic LDL receptor deficiency (LDLr-/-) mice with anti-miR-33 restored defective autophagy in macrophage foam cells and plaques. Ramirez CM et al. [61] showed that miR-758 repressed the expression of ABCA1, on the contrary, the inhibition of miR-758 increased ABCA1 expression. According to Liang B et al. [62], miR-20a/b inhibited ABCA1 expression, decreased cholesterol efflux and raised cholesterol content in THP-1 and RAW 264.7 macrophage-derived foam cells, and these effects could be reversed by miR-20a/b inhibitors. Lv YC et al. [63] presented that miR-19b suppressed ABCA1-dependent cholesterol efflux. Svenja Meiler et al. [64] found that long-term in vivo administration of anti-miRNA-302a increased the expression of $\mathrm{ABCA} 1$ in the liver but reduced the atherosclerotic lesions in atherogenic diet fed $\mathrm{LDLr}^{-/}$mice.

\section{Interplay of Exosomal and Autophagic Pathways, and CVDs}

There were two main pathways regulating autophagy, namely, PI3K-Akt-mammalian rapamycin target protein (mTOR) and AMPK-mTOR [65]. PI3K/Akt and mTOR activation are responsible for downregulated autophagy, and AMPK activation inhibits mTOR contributing to upregulated autophagy likewise the function of rapamycin [65]. Currently, the crosstalk between exosomal and autophagic pathways has been reported in a growing number of diseases. The effects of exosomal process on autophagic activity are not completely consistent, maybe partly since the regulation effects of exosomes-related non-coding RNAs on the autophagic signaling pathways varies among the current studies. According to Li L et al. [66], overexpression of miR-221/222 inhibited the level of PTEN and activated Akt signaling, and subsequently reduced the expression of hallmarks that positively relate to autophagy including LC3II, ATG5 and Beclin1, and increased the expression of SQSTM1/p62. MiR-221/222 from human aortic smooth muscle cells (HAoSMCs)derived exosomes, inhibited autophagy in HUVECs by modulating PTEN/Akt signaling pathway. The cardioprotective effects of EnMSCs are probably owing to reduced expression of PTEN and increased phosphorylation of Akt by stimulation of exosomal miR21[36]. In addition, Suzuki T et al. [67] also showed that MSC- exosomes decreased autophagy by regulation of mTOR and autophagy-associated protein-13 (ATG13). Liu X et al. [68] revealed that miRNA-223 attenuated hypoxia-induced apoptosis and excessive autophagy in neonatal rat cardiomyocytes and $\mathrm{H} 9 \mathrm{C} 2$ cells, via 
Akt/mTOR pathway by targeting poly(ADP-ribose) polymerase 1 (PARP-1). Similarly, Arslan F et al.[31]showed that MCS-exo exerts cardio-protection effect mediated by activation of PI3K/Akt pathway. miR130a and miR-214 attenuate cardiac dysfunction by activation of PI3K/Akt signaling and downregulation of PTEN expression $[69,70]$. However, according to Liang Liu et al. [71], treated $\mathrm{H} 9 \mathrm{C} 2$ cells with MSCs-derived exosomes enhanced autophagy via AMPK/mTOR and Akt/mTOR pathways. In vivo, exosomes reduced apoptosis and the myocardial infarct size in rats underwent ischemic/reperfusion injury by upregulating LC3B expression. Yang $\mathrm{Y}$ et al. [29] reported that, inhibition of exosomal miR-30a could attenuate apoptosis of cardiomyocytes induced by hypoxia via augmentation of autophagy, characterized by enhanced expression of core autophagy regulators beclin-1, Atg12, and LC3II/I, suggesting a novel pathway of autophagy in cardiomyocytes after hypoxia.

\section{Conclusions and Prospective}

Autophagy and exosomes maintain cell homeostasis and fitness in a coordinated manner by removal or secretion harmful substance. The crosstalk between autophagic and exosomal pathways, and the property of exosomes and drug nanocarriers make the donation of exosomes as a cardio-protective approach along with the therapeutic potential for cardiac regeneration [72, 73, 74]. For the evidences that autophagy is an emerging target for CVDs, and tradition Chinese herbal medicines have been demonstrated to be beneficial in treating CVDs through modulation of autophagy $[46,50,52,75,76]$. Hence, it is supposed that the MSCs-derived exosomes loaded with traditional Chinese medicine supplement could manipulate medicine-targeting autophagy, a promising therapeutic approach for CVDs [77]. On the other hand, the therapeutic potential of MSCs-derived eoxomes makes the possibility to use exosomes in-situ tissue regeneration and remodeling [78-81].

\section{Conflicts of interest}

The authors have no conflicts of interests to declare.

\section{Acknowledgement}

The study was supported by Beijing NOVA Program (No. Z171100001117027).

\section{References}

[1] Su SA, Xie Y, Fu Z, Wang Y, Wang JA, Xiang M (2017). Emerging role of exosome-mediated intercellular communication in vascular remodeling. Oncotarget, 8: 25700-12.

[2] Lu M, Yuan S, Li S, Li L, Liu M, Wan S (2018). The Exosome-Derived Biomarker in Atherosclerosis and Its Clinical Application. J Cardiovasc Transl Res. doi: 10.1007/s12265-018-9796-y. [Epub ahead of print]

[3] $\mathrm{Lu} \mathrm{X}$. The Role of Exosomes and Exosome-derived microRNAs in Atherosclerosis (2017). Curr Pharm Des, 23: 6182-93.

[4] Vindis C. Autophagy: an emerging therapeutic target in vascular diseases (2015). Br J Pharmacol, 172: 2167-78.

[5] Luo Z, Xu W, Ma S, Qiao H, Gao L, Zhang R, et al (2017). Moderate Autophagy Inhibits Vascular Smooth Muscle Cell Senescence to Stabilize Progressed Atherosclerotic Plaque via the mTORC1/ULK1/ATG13 Signal Pathway. Oxid Med Cell Longev, 2017: 3018190.

[6] Baixauli F, López-Otín C, Mittelbrunn M (2014). Exosomes and autophagy: coordinated mechanisms for the maintenance of cellular fitness. Front Immunol, 5: 403.

[7] Desdín-Micó G, Mittelbrunn M (2017). Role of exosomes in the protection of cellular homeostasis. Cell Adh Migr, 11: 127-34.

[8] Jiang M, Wang H, Jin M, Yang X, Ji H, Jiang Y, et al (2018). Exosomes from MiR-30d-5p-ADSCs Reverse Acute Ischemic Stroke-Induced, Autophagy-Mediated Brain Injury by Promoting M2 Microglial/Macrophage Polarization. Cell Physiol Biochem, 47: 864-78.

[9] Abdulrahman BA, Abdelaziz DH, Schatzl HM (2018). Autophagy regulates exosomal release of prions in neuronal cells. J Biol Chem, 293: 8956-68.

[10] Ojha CR, Lapierre J, Rodriguez M, Dever SM, Zadeh MA, DeMarino C, et al (2017). Interplay between Autophagy, Exosomes and HIV-1 Associated Neurological Disorders: New Insights for Diagnosis and Therapeutic Applications. Viruses, 9. pii: E176.

[11] Liu J, Jiang M, Deng S, Lu J, Huang H, Zhang Y, et al (2018). miR-93-5p-Containing Exosomes Treatment Attenuates Acute Myocardial Infarction-Induced Myocardial Damage. Mol Ther Nucleic Acids, 11: 103-15.

[12] Qu Y, Zhang Q, Cai X, Li F, Ma Z, Xu M, et al (2017). Exosomes derived from miR-181-5p-modified adiposederived mesenchymal stem cells prevent liver fibrosis via autophagy activation. J Cell Mol Med, 21: 2491-502.

[13] Zhang Y, Hu YW, Zheng L, Wang Q (2017). Characteristics and Roles of Exosomes in Cardiovascular Disease. DNA Cell Biol, 36: 202-11.

[14] Emanueli C, Shearn AI, Angelini GD, Sahoo S (2015). Exosomes and exosomal miRNAs in cardiovascular protection and repair. Vascul Pharmacol, 71: 24-30.

[15] Huang C, Huang Y, Zhou Y, Nie W, Pu X, Xu X, et al (2018). Exosomes derived from oxidized LDL-stimulated macrophages attenuate the growth and tube formation of endothelial cells. Mol Med Rep, 17: 4605-10.

[16] Chen L, Yang W, Guo Y, Chen W, Zheng P, Zeng J, et al (2017). Exosomal lncRNA GAS5 regulates the apoptosis of macrophages and vascular endothelial cells in atherosclerosis. PLoS One, 12: e0185406.

[17] Hergenreider E, Heydt S, Tréguer K, Boettger T, Horrevoets AJ, Zeiher AM, et al (2012). Atheroprotective communication between endothelial cells and smooth muscle cells through miRNAs. Nat Cell Biol, 14: 249-56.

[18] Zheng B, Yin WN, Suzuki T, Zhang XH, Zhang Y, Song LL, et al (2017). Exosome-Mediated miR-155 Transfer 
from Smooth Muscle Cells to Endothelial Cells Induces Endothelial Injury and Promotes Atherosclerosis. Mol Ther, 25: 1279-94.

[19] Li J, Tan M, Xiang Q, Zhou Z, Yan H (2017). Thrombinactivated platelet-derived exosomes regulate endothelial cell expression of ICAM-1 via microRNA-223 during the thrombosis-inflammation response. Thromb Res, 154: 96105.

[20] Tan M, Yan HB, Li JN, Li WK, Fu YY, Chen W, et al (2016). Thrombin Stimulated Platelet-Derived Exosomes Inhibit Platelet-Derived Growth Factor Receptor-Beta Expression in Vascular Smooth Muscle Cells. Cell Physiol Biochem, 38: 2348-65.

[21] Wang F, Chen FF, Shang YY, Li Y, Wang ZH, Han L, et al (2018). Insulin resistance adipocyte-derived exosomes aggravate atherosclerosis by increasing vasa vasorum angiogenesis in diabetic ApoE(-/-) mice. Int $\mathrm{J}$ Cardiol, 265:181-7.

[22] Gao W, Liu H, Yuan J, Wu C, Huang D, Ma Y, et al (2016). Exosomes derived from mature dendritic cells increase endothelial inflammation and atherosclerosis via membrane TNF-alpha mediated NF-kappaB pathway. J Cell Mol Med, 20: 2318-27.

[23] Hu G, Drescher KM, Chen XM (2012). Exosomal miRNAs: Biological Properties and Therapeutic Potential. Front Genet, 3: 56.

[24] Sayed AS, Xia K, Yang TL, Peng J (2013). Circulating microRNAs: a potential role in diagnosis and prognosis of acute myocardial infarction. Dis Markers, 35: 561-6.

[25] Corsten MF, Dennert R, Jochems S, Kuznetsova T, Devaux Y, Hofstra L, et al (2010). Circulating MicroRNA-208b and MicroRNA-499 reflect myocardial damage in cardiovascular disease. Circ Cardiovasc Genet, 3: 499-506.

[26] Li Y, Lu J, Bao X, Wang X, Wu J, Li X (2016). MiR-499$5 p$ protects cardiomyocytes against ischaemic injury via anti-apoptosis by targeting PDCD4. Oncotarget, 7: 3560717.

[27] Wang GK, Zhu JQ, Zhang JT, Li Q, Li Y, He J, et al (2010). Circulating microRNA: a novel potential biomarker for early diagnosis of acute myocardial infarction in humans. Eur Heart J, 31: 659-66.

[28] Olivieri F, Antonicelli R, Lorenzi M, D'Alessandra Y, Lazzarini R, Santini G, et al (2013). Diagnostic potential of circulating miR-499-5p in elderly patients with acute nonST-elevation myocardial infarction. Int J Cardiol, 167: 5316.

[29] Yang Y, Li Y, Chen X, Cheng X, Liao Y, Yu X (2016). Exosomal transfer of miR-30a between cardiomyocytes regulates autophagy after hypoxia. J Mol Med (Berl), 94: 711-24.

[30] Cortez-Dias N, Costa MC, Carrilho-Ferreira P, Silva D, Jorge C, Calisto C, et al (2016). Circulating miR-1225p/miR-133b Ratio Is a Specific Early Prognostic Biomarker in Acute Myocardial Infarction. Circ J, 80: 218391.

[31] Arslan F, Lai RC, Smeets MB, Akeroyd L, Choo A, Aguor EN, et al (2013). Mesenchymal stem cell-derived exosomes increase ATP levels, decrease oxidative stress and activate PI3K/Akt pathway to enhance myocardial viability and prevent adverse remodeling after myocardial ischemia/reperfusion injury. Stem Cell Res, 10: 301-12.

[32] Lai RC, Arslan F, Lee MM, Sze NS, Choo A, Chen TS, et al (2010). Exosome secreted by MSC reduces myocardial ischemia/reperfusion injury. Stem Cell Res, 4: 214-22.

[33] Teng X, Chen L, Chen W, Yang J, Yang Z, Shen Z (2015). Mesenchymal Stem Cell-Derived Exosomes Improve the Microenvironment of Infarcted Myocardium Contributing to Angiogenesis and Anti-Inflammation. Cell Physiol Biochem, 37: 2415-24.

[34] Zhang Z, Yang J, Yan W, Li Y, Shen Z, Asahara T (2016). Pretreatment of Cardiac Stem Cells With Exosomes Derived From Mesenchymal Stem Cells Enhances Myocardial Repair. J Am Heart Assoc, 5. pii: e002856.

[35] Wang XL, Zhao YY, Sun L, Shi Y, Li ZQ, Zhao XD, et al (2018). Exosomes derived from human umbilical cord mesenchymal stem cells improve myocardial repair via upregulation of Smad7. Int J Mol Med, 41: 3063-72.

[36] Wang K, Jiang Z, Webster KA, Chen J, Hu H, Zhou Y, et al (2017). Enhanced Cardioprotection by Human Endometrium Mesenchymal Stem Cells Driven by Exosomal MicroRNA-21. Stem Cells Transl Med, 6: 20922.

[37] Luo Q, Guo D, Liu G, Chen G, Hang M, Jin M (2017). Exosomes from MiR-126-Overexpressing Adscs Are Therapeutic in Relieving Acute Myocardial Ischaemic Injury. Cell Physiol Biochem, 44: 2105-16.

[38] Yuan M, Zhang L, You F, Zhou J, Ma Y, Yang F, et al (2017). MiR-145-5p regulates hypoxia-induced inflammatory response and apoptosis in cardiomyocytes by targeting CD40. Mol Cell Biochem, 431: 123-31.

[39] Vicencio JM, Yellon DM, Sivaraman V, Das D, Boi-Doku C, Arjun S, et al (2015). Plasma exosomes protect the myocardium from ischemia-reperfusion injury. J Am Coll Cardiol, 65: 1525-36.

[40] Chen L, Wang Y, Pan Y, Zhang L, Shen C, Qin G, et al (2013). Cardiac progenitor-derived exosomes protect ischemic myocardium from acute ischemia/reperfusion injury. Biochem Biophys Res Commun, 431: 566-71.

[41] Xiao J, Pan Y, Li XH, Yang XY, Feng YL, Tan HH, et al (2016). Cardiac progenitor cell-derived exosomes prevent cardiomyocytes apoptosis through exosomal miR-21 by targeting PDCD4. Cell Death Dis, 7: e2277.

[42] de Gonzalo-Calvo D, van der Meer RW, Rijzewijk LJ, Smit JW, Revuelta-Lopez E, Nasarre L, et al (2017). Serum microRNA-1 and microRNA-133a levels reflect myocardial steatosis in uncomplicated type 2 diabetes. Sci Rep, 7: 47.

[43] Wang X, Gu H, Huang W, Peng J, Li Y, Yang L, et al (2016). Hsp20-Mediated Activation of Exosome Biogenesis in Cardiomyocytes Improves Cardiac Function and Angiogenesis in Diabetic Mice. Diabetes, 65: 3111-28.

[44] Gatica D, Chiong M, Lavandero S, Klionsky DJ (2015). Molecular mechanisms of autophagy in the cardiovascular system. Circ Res, 116: 456-67.

[45] Schiattarella GG, Hill JA (2016). Therapeutic targeting of autophagy in cardiovascular disease. J Mol Cell Cardiol, 95: 86-93.

[46] Wang D, Yu W, Liu Y, Zhong G, Zhao Z, Yan X, et al (2017). Roles of Autophagy in Ischemic Heart Diseases and the Modulatory Effects of Chinese Herbal Medicine. Am J Chin Med, 45: 1401-19.

[47] Rayner KJ, Moore KJ (2014). MicroRNA control of highdensity lipoprotein metabolism and function. Circ Res, 114: 183-92.

[48] Zhai H, Fesler A, Ju J (2013). MicroRNA: a third dimension in autophagy. Cell Cycle, 12: 246-50.

[49] Peng S, Xu LW, Che XY, Xiao QQ, Pu J, Shao Q, et al 
(2018). Atorvastatin Inhibits Inflammatory Response, Attenuates Lipid Deposition, and Improves the Stability of Vulnerable Atherosclerotic Plaques by Modulating Autophagy. Front Pharmacol, 9: 438.

[50] Chen Y, Li M, Zhang Y, Di M, Chen W, Liu X, et al (2018). Traditional Chinese medication Tongxinluo attenuates apoptosis in ox-LDL-stimulated macrophages by enhancing Beclin-1-induced autophagy. Biochem Biophys Res Commun, 501: 336-42.

[51] Sun W, Lin Y, Chen L, Ma R, Cao J, Yao J, et al (2018). Legumain suppresses OxLDL-induced macrophage apoptosis through enhancement of the autophagy pathway. Gene, 652: 16-24.

[52] Yang P, Ling L, Sun W, Yang J, Zhang L, Chang G, et al (2018). Ginsenoside Rg1 inhibits apoptosis by increasing autophagy via the AMPK/mTOR signaling in serum deprivation macrophages. Acta Biochim Biophys Sin (Shanghai), 50: 144-55.

[53] Ning H, Liu D, Yu X, Guan X (2017). Oxidized low-density lipoprotein-induced p62/SQSTM1 accumulation in THP-1derived macrophages promotes IL-18 secretion and cell death. Exp Ther Med, 14: 5417-23.

[54] Shao BZ, Han BZ, Zeng YX, Su DF, Liu C (2016). The roles of macrophage autophagy in atherosclerosis. Acta Pharmacol Sin, 37: 150-6.

[55] Zhang Q, Yang YJ, Wang H, Dong QT, Wang TJ, Qian HY, et al (2012). Autophagy activation: a novel mechanism of atorvastatin to protect mesenchymal stem cells from hypoxia and serum deprivation via AMP-activated protein kinase/mammalian target of rapamycin pathway. Stem Cells Dev, 21: 1321-32.

[56] Rayner KJ, Suárez Y, Dávalos A, Parathath S, Fitzgerald ML, Tamehiro N, et al (2010). MiR-33 contributes to the regulation of cholesterol homeostasis. Science, 328: 15703.

[57] Najafi-Shoushtari SH, Kristo F, Li Y, Shioda T, Cohen DE, Gerszten RE, et al (2010). MicroRNA-33 and the SREBP host genes cooperate to control cholesterol homeostasis. Science, 328: 1566-9.

[58] Horie T, Baba O, Kuwabara Y, Chujo Y, Watanabe S, Kinoshita M, et al (2012). MicroRNA-33 deficiency reduces the progression of atherosclerotic plaque in ApoE/- mice. J Am Heart Assoc, 1: e003376.

[59] Rayner KJ, Sheedy FJ, Esau CC, Hussain FN, Temel RE, Parathath S, et al (2011). Antagonism of miR-33 in mice promotes reverse cholesterol transport and regression of atherosclerosis. J Clin Invest, 121: 2921-31.

[60] Ouimet M, Ediriweera H, Afonso MS, Ramkhelawon B, Singaravelu R, Liao X, et al (2017). microRNA-33 Regulates Macrophage Autophagy in Atherosclerosis. Arterioscler Thromb Vasc Biol, 37: 1058-67.

[61] Ramirez CM, Dávalos A, Goedeke L, Salerno AG, Warrier N, Cirera-Salinas D, et al (2011). MicroRNA-758 regulates cholesterol efflux through posttranscriptional repression of ATP-binding cassette transporter A1. Arterioscler Thromb Vasc Biol, 31: 2707-14.

[62] Liang B, Wang X, Song X, Bai R, Yang H, Yang Z, et al (2017). MicroRNA-20a/b regulates cholesterol efflux through post-transcriptional repression of ATP-binding cassette transporter A1. Biochim Biophys Acta, 1862: 92938.

[63] Lv YC, Tang YY, Peng J, Zhao GJ, Yang J, Yao F, et al (2014). MicroRNA-19b promotes macrophage cholesterol accumulation and aortic atherosclerosis by targeting ATPbinding cassette transporter A1. Atherosclerosis, 236: 21526.

[64] Meiler S, Baumer Y, Toulmin E, Seng K, Boisvert WA (2015). MicroRNA 302a is a novel modulator of cholesterol homeostasis and atherosclerosis. Arterioscler Thromb Vasc Biol, 35: 323-31.

[65] Parzych KR, Klionsky DJ (2014). An overview of autophagy: morphology, mechanism, and regulation. Antioxid Redox Signal, 20: 460-73.

[66] Li L, Wang Z, Hu X, Wan T, Wu H, Jiang W, et al (2016). Human aortic smooth muscle cell-derived exosomal miR221/222 inhibits autophagy via a PTEN/Akt signaling pathway in human umbilical vein endothelial cells. Biochem Biophys Res Commun, 479: 343-50.

[67] Suzuki T, Inoki K (2011). Spatial regulation of the mTORC1 system in amino acids sensing pathway. Acta Biochim Biophys Sin (Shanghai), 43: 671-9.

[68] Liu X, Deng Y, Xu Y, Jin W, Li H (2018). MicroRNA-223 protects neonatal rat cardiomyocytes and $\mathrm{H} 9 \mathrm{c} 2$ cells from hypoxia-induced apoptosis and excessive autophagy via the Akt/mTOR pathway by targeting PARP-1. J Mol Cell Cardiol, 118: 133-46.

[69] Lu C, Wang X, Ha T, Hu Y, Liu L, Zhang X, et al (2015). Attenuation of cardiac dysfunction and remodeling of myocardial infarction by microRNA-130a are mediated by suppression of PTEN and activation of PI3K dependent signaling. J Mol Cell Cardiol, 89: 87-97.

[70] Wang X, Ha T, Hu Y, Lu C, Liu L, Zhang X, et al (2016). MicroRNA-214 protects against hypoxia/reoxygenation induced cell damage and myocardial ischemia/reperfusion injury via suppression of PTEN and Bim1 expression. Oncotarget, 7: 86926-36.

[71] Liu L, Jin X, Hu CF, Li R, Zhou Z, Shen CX (2017). Exosomes Derived from Mesenchymal Stem Cells Rescue Myocardial Ischaemia/Reperfusion Injury by Inducing Cardiomyocyte Autophagy Via AMPK and Akt Pathways. Cell Physiol Biochem, 43: 52-68.

[72] Lai RC, Yeo RW, Tan KH, Lim SK (2013). Exosomes for drug delivery - a novel application for the mesenchymal stem cell. Biotechnol Adv, 31: 543-51.

[73] Garrido V, Mendoza-Torres E, Riquelme JA, Díaz A, Pizarro M, Bustamante M, et al (2017). Novel Therapies Targeting Cardioprotection and Regeneration. Curr Pharm Des, 23: 2592-615.

[74] Papandreou ME, Tavernarakis N (2017). Autophagy and the endo/exosomal pathways in health and disease. Biotechnol $\mathrm{J}, 12$.

[75] Qiao L, Zhang X, Liu M, Liu X, Dong M, Cheng J, et al. Corrigendum: Ginsenoside Rb1 Enhances Atherosclerotic Plaque Stability by Improving Autophagy and Lipid Metabolism in Macrophage Foam Cells. Front Pharmacol, 2017. 8: 964.

[76] Zhang BC, Zhang CW, Wang C, Pan DF, Xu TD, Li DY (2016). Luteolin Attenuates Foam Cell Formation and Apoptosis in Ox-LDL-Stimulated Macrophages by Enhancing Autophagy. Cell Physiol Biochem, 39: 2065-76.

[77] Sun D, Zhuang X, Xiang X, Liu Y, Zhang S, Liu C, et al (2010). A novel nanoparticle drug delivery system: the antiinflammatory activity of curcumin is enhanced when encapsulated in exosomes. Mol Ther, 18: 1606-14.

[78] Vizoso FJ, Eiro N, Cid S, Schneider J, Perez-Fernandez R (2017). Mesenchymal Stem Cell Secretome: Toward Cell- 
Free Therapeutic Strategies in Regenerative Medicine. Int J Mol Sci, 18. pii: E1852.

[79] De Jong OG, Van Balkom BW, Schiffelers RM, Bouten CV, Verhaar MC (2014). Extracellular vesicles: potential roles in regenerative medicine. Front Immunol, 5: 608.

[80] Lamichhane TN, Sokic S, Schardt JS, Raiker RS, Lin JW, Jay SM (2015). Emerging roles for extracellular vesicles in tissue engineering and regenerative medicine. Tissue Eng Part B Rev, 21: 45-54.

[81] Nazimek K, Bryniarski K, Santocki M, Ptak W (2015). Exosomes as mediators of intercellular communication: clinical implications. Pol Arch Med Wewn, 125: 370-80. 